\title{
A Probabilistic Multidimensional Scaling Vector Model
}

\author{
Wayne S. DeSarbo and Richard L. Oliver \\ University of Pennsylvania \\ Geert De Soete \\ University of Ghent
}

\begin{abstract}
This article presents the development of a new stochastic multidimensional scaling (MDS) method, which operates on paired comparisons data and renders a spatial representation of subjects and stimuli. Subjects are represented as vectors and stimuli as points in a $T$ dimensional space, where the scalar products, or projections of the stimulus points onto the subject vectors, provide respective information as to the utility (or whatever latent construct is under investigation) of the stimuli to the subjects. The psychometric literature
\end{abstract}

\begin{abstract}
concerning related MDs methods that also operate on paired comparisons data is reviewed, and a technical description of the new method is provided. A small monte carlo analysis performed on synthetic data with the new method is also presented. To illustrate the versatility of the model, an application measuring consumer satisfaction and investigating the impact of hypothesized determinants, using one of the optional reparameterized models, is described. Future areas of further research are identified.
\end{abstract}

The method of paired comparisons typically involves the presentation of pairs of stimuli to one or more subjects. The basic experimental unit is the comparison of two stimuli, $A$ and $B$, by a single subject who, in the simplest case, must choose between them (David, 1963). This method was introduced by Fechner (1860) with considerable extensions made popular by Thurstone (1927). The method of paired comparisons is gainfully used in situations in which the stimuli to be compared can be judged only subjectively; that is, when it is impossible to make relevant continuous measurement in order to decide which of two stimuli are preferable. The most frequent applications have been generally for taste testing, consumer tests, color comparisons, personnel ratings, and choice behavior (David, 1963). For $J$ stimuli and $I$ judges, the total number of paired comparisons will be $I\left(\begin{array}{l}J \\ 2\end{array}\right)$, though a number of incomplete designs are also available (cf. Bock \& Jones, 1968; Box, Hunter, \& Hunter, 1978) for reducing the total number of pairwise judgments under simplifying sets of assumptions. Note that when $J$ is large, the task of making consistent pairwise judgments becomes quite difficult. Oftentimes, intransitivities or circular triads occur in such data where, for example, $A$ may be preferred to $B$, and $B$ preferred to $C$, but the same subject claims to prefer $C$ to $A$. Therefore, probabilistic models are needed to analyze such paired comparisons data.

There have been a number of unidimensional scaling procedures proposed to obtain scale values for stimuli from (aggregated) paired comparisons data (Bock \& Jones, 1968; Thurstone, 1927; Torgerson,

APPLIED PSYCHOLOGICAL MEASUREMENT

Vol. 10, No. 1, March 1986, pp. 79-98

(c) Copyright 1986 Applied Psychological Measurement Inc.

0146-6216/86/010079-20\$2.25 
1958). More recently, multidimensional scaling (MDS) models have been devised to account for the multidimensional nature of the stimuli. One option in the computer program MDPREF (Carroll, 1980), which typically operates on two-way, two-mode dominance or profile data, is to collapse the paired comparison matrix for each subject into a vector of marginals, and then analyze the resulting two-way, two-mode matrix of (dominating) integer counts. Unfortunately, there is a substantial loss of information involved in collapsing such individual paired comparison matrices to perform this analysis.

Bechtel, Tucker, and Chang (1971) developed a scalar products model (Slater, 1960; Tucker, 1960) for examining graded paired comparisons responses (i.e., when respondents indicate which of two stimuli is preferred and to what extent). They impose restrictions on sums and variances, and constraints on various parameters to insure uniqueness of the solution (e.g., orthogonality). In addition, Schonemann and Wang (1972) have presented an unfolding methodology for paired comparisons data as a generalization of the Bradley-Terry-Luce model (cf. Luce, 1959). Zinnes and Wolff (1977) have developed a probabilistic multidimensional Thurstonian model for spatially representing the structure in different-same judgments using a threshold parameter. Cooper and Nakanishi (1983) have developed two logit models (vector and ideal point) for the external analysis of paired comparisons data.

Recently, Carroll (1980) suggested use of the wandering vector model for the analysis of paired comparisons data. According to the vector model, it is assumed that each subject can be represented by a vector and that individual subjects will prefer that stimulus from a pair having the largest projection on that vector. The direction cosines of this vector specify the relative weights the subject attaches to the underlying dimensions. The wandering vector model assumes that a subject's vector wanders or fluctuates from a central vector in such a way that the distribution of the vector termini is multivariate normal. De Soete and Carroll (1983) developed a maximum likelihood method for fitting this model and proposed various extensions of the original Carroll model to accommodate additional sources of error, graded paired comparisons, and stimulus restrictions.

Unfortunately, the De Soete and Carroll (1983) model requires replications of paired comparison matrices per subject to estimate more than one vector. This turns out to be a rather difficult task in terms of data collection. Without such replications, only one centroid vector can be estimated for a sample of I judges. Assuming considerable heterogeneity in the sample, the single centroid vector may be estimated with considerably high variances on the terminus. In addition, no provision is available to explore individual differences (with replications) as a function of specified subject differences (such as demographic characteristics).

This article proposes an alternative probabilistic vector MDS model that operates on paired comparisons. The present model can estimate separate subject vectors without requiring within-subject replications. A variety of possible model specifications are provided in which vectors and/or stimuli can be reparameterized as a function of specified background variables. The model structure is described as well as its program options, and a small monte carlo analysis with a synthetic data set is provided. Next, an application is provided whereby consumer satisfaction is measured and the relative impact of four hypothesized determinants is studied. Finally, further research avenues are discussed.

\section{Methodology}

\section{Research Objectives}

The objective of this research was to develop a new MDS methodology that operates on paired comparison judgments so that subjects and stimuli can be displayed spatially, thus permitting inferences concerning the nature of the latent unobservable construct under investigation (e.g., preference, choice, 
utility, satisfaction, etc.). In doing so, two subobjectives were addressed. The first concerned the capability to investigate the nature of individual differences on the latent construct and its measurement, whereas the second involved modeling the effect of specific stimulus features (attributes) on the measurement of the latent construct.

\section{The Model}

Let: $i=1, \ldots, I$ subjects,

$j, k=1, \ldots, J$ stimuli,

$t=1, \ldots, T$ dimensions in an MDs context,

$l=1, \ldots, L$ stimulus features or attributes,

$n=1, \ldots, N$ subject descriptor variables (e.g., socioeconomic background variables),

$X_{i j}=$ the $j$ th stimulus presented to the $i$ th subject,

$\delta_{i j k}=\left\{\begin{array}{l}1 \text { if subject } i \text { finds } X_{i j} \text { more satisfying than } X_{i k}, \\ 0 \text { otherwise, }\end{array}\right.$

$H_{j l}=$ the $l$ th feature/attribute value for the $j$ th stimulus,

$Y_{i n}=$ the $n$th variable value for the $i$ th subject,

$a_{i t}=$ the $t$ th coordinate for subject $i$,

$b_{j t}=$ the $t$ th coordinate for stimulus $j$,

$\alpha_{n t}=$ the impact coefficient of the $n$th subject descriptor variable on the th dimension, and

$\gamma_{t t}=$ the impact coefficient of the $l$ th stimulus feature/attribute on the $t$ th dimension.

Now, define a latent subject satisfaction construct:

$\dot{V}_{i j}=U_{i j}+e_{i j}$,

where: $V_{i j}=$ the (latent) satisfaction of stimulus $j$ to subject $i$,

$$
\begin{aligned}
U_{i j} & =\sum_{t=1}^{T} a_{i l} b_{j t}, \\
e_{i j} & =\text { error. }
\end{aligned}
$$

Here, $U_{i j}$ refers to a "true" utility or latent satisfaction score for subject $i$ concerning object $j$. It is modeled as equal to the scalar product of the stimulus coordinates $\left(b_{j t}\right)$ and the subject vector $\left(a_{i t}\right)$. The order of utility or satisfaction for a given subject is thus assumed to be given by the projection of the stimuli onto the vector representing that subject.

As with other companion vector MDS models (e.g., MDPREF, factor analysis, PREFMAP - Model 4, wandering vector model, etc.), the present model assumes that utility or satisfaction changes monotonically with all dimensions. That is, it assumes that if a certain amount of a given quantity is good (bad), even more must be better (worse). This implies that the "iso-utility" or "iso-satisfaction" contours for the vector model comprise a family of parallel straight lines perpendicular to the particular vector. Here, too, one way of interpreting vectors in this model is in terms of the relative importance of the dimensions to utility or satisfaction. The cosines of the angles a vector forms with the coordinate axes directly measure these relative importances. These "importance weights" act like coefficients in a linear combination of dimensions.

It is assumed that

$e_{i j} \sim N\left(0, \sigma_{i}^{2}\right) \quad$ (where $\sigma_{i}^{2}$ is the variance parameter for the $i$ th subject) ,

$\operatorname{Cov}\left(e_{i j}, e_{i k}\right)=0$, for all $i, j \neq k$,

$\operatorname{Cov}\left(e_{i j}, e_{i^{\prime} k}\right)=0$, for all $i \neq i^{\prime}, j, k$. 
Suppose that subject $i$ is presented two stimuli (e.g., products or brands) $j$ and $k$ and is asked to select the one that is "more satisfying." Then,

$$
\begin{aligned}
P\left(\delta_{i j k}=1\right) & =P\left(V_{i j}>V_{i k}\right)=P\left[e_{i k}-e_{i j}<\sum_{t=1}^{T} a_{i r}\left(b_{j t}-b_{k t}\right)\right] \\
& =\Phi\left[\frac{\sum_{t=1}^{T} a_{i t}\left(b_{j t}-b_{k t}\right)}{\left(2 \sigma_{i}^{2}\right)^{1 / 2}}\right]=\Phi\left[\sum_{t=1}^{T} a_{i t}\left(b_{j t}-b_{k t}\right)\right],
\end{aligned}
$$

where $\Phi$ denotes the standard normal distribution function integrated over the interval from $-\infty$ to $\sum_{t} a_{i t}\left(b_{j t}-b_{k t}\right)$. Note that the $\sigma_{i}^{2}$ s are dropped in Equation 3, since the $\sigma_{i} s$ are confounded with the estimation of the $a_{i t}$ s (i.e., the $a_{i l}$ s can absorb the denominator terms). Similarly,

$P\left(\delta_{i j k}=0\right)=1-\Phi\left[\frac{\sum_{i=1}^{T} a_{i t}\left(b_{j t}-b_{k t}\right)}{\left(2 \sigma_{i}^{2}\right)^{1 / 2}}\right]=1-\Phi\left[\sum_{t=1}^{T} a_{i t}\left(b_{j t}-b_{k t}\right)\right]$.

The general form of the likelihood function, assuming independence over subscripts $i, j$, and $k$, is

$L=\prod_{i=1}^{I}\left\{\prod_{j<k}^{J} \Phi(\cdot)^{\delta_{j k}} \prod_{j<k}^{J} \amalg[1-\Phi(\cdot)]^{1-\delta_{i j k}}\right\}$,

where

$\Phi(\cdot)=\Phi\left[\sum_{t=1}^{T} a_{i t}\left(b_{j t}-b_{k t}\right)\right]$

Taking logs, the log likelihood function is obtained,

$\ln L=\sum_{i=1}^{I} \sum_{j<k}^{J} \sum \delta_{i j k} \ln \Phi(\cdot)+\sum_{i=1}^{I} \sum_{j<k}^{J}\left\{\left[1-\delta_{i j k}\right] \ln [1-\Phi(\cdot)]\right\}$.

Maximum likelihood procedures are used to estimate $\mathbb{A}=\left\|a_{i t}\right\|$ and $\mathbb{B}=\left\|b_{j i t}\right\|$, given $\Delta=\left\|\delta_{i j k}\right\|$ and $T$. It should be noted that the procedure is not restricted to produce orthogonal dimensions in $\mathbb{A}, \mathbb{B}$, or scores $\mathbb{U}=|| U_{i j} \mid$. In general, the extracted dimensions will be oblique, as will the latent projection scores.

Unlike Carroll's (1980) original formulation of the wandering vector model, the model proposed here posits no explicit distribution on the subject vectors. Rather, it is a type of random utility model (Thurstone, 1927), in which the latent construct being modeled (e.g., utility, preference, satisfaction, etc.) is specified following McFadden (1976), with the introduction of an error term. De Soete and Carroll (1983) mentioned a similar extension to their maximum likelihood method for fitting the Carroll (1980) wandering vector model. However, they require replications of paired comparisons data for each subject in order to estimate more than a single vector, since the subject vector is modeled as being explicitly normally distributed. However, the wandering vector model has the advantage of implying only moderate stochastic transitivity, whereas the present model, like Thurstone's (1927) Law of Comparative Judgment Case V, implies strong stochastic transitivity. Therefore, this new proposed methodology may not be appropriate in situations in which there are serious violations to strong stochastic transitivity. Monte carlo analyses are required to investigate the point at which such violations begin to affect the derived solutions.

\section{Program Options}

The probabilistic vector model developed here can accommodate a number of different model specifications and options. Users can perform either an internal analysis (when the user estimates both 
points and vectors) or an external analysis (when the user can fix one or more sets of coordinates throughout the analysis). Users can also select among a number of methods of generating starting estimates, including a user-defined option. Also, since the scalar products model is invariant to particular nonsingular transformations, options are provided to rotate either $\mathbf{A}$ or $\mathbf{B}$ to principal axes for possible enhancement of interpretation.

Perhaps the most valuable program options concern the possibility of reparameterizing subject vector coordinates and/or stimulus coordinates as functions of prespecified background features or attributes. That is, a user may reparameterize subject vectors using

$a_{i t}=\sum_{n=1}^{N} Y_{i n} \alpha_{n t}$

and/or stimulus coordinates using

$b_{j t}=\sum_{l=1}^{L} H_{j l} \gamma_{l t}$.

As in CANDElinc (Carroll, Pruzansky, \& Kruskal, 1980), Three-Way Multivariate Conjoint Analysis (DeSarbo, Carroll, Lehmann, \& O'Shaughnessy, 1982), and GENFOLD2 (DeSarbo \& Rao, 1984), these reparameterization options can be used to examine what impact such features/attributes have on the derived solution. This can often aid in interpreting the resulting solution. Note that, because of potential problems associated with placing such restrictions on subject vectors as discussed in Carroll et al. (1981) and DeSarbo et al. (1982), an option exists to estimate a multiplicative stretching/shrinking parameter, $\tau_{i}$, on the righthand side of Equation 8 . These $\tau_{i}$ parameters are multiplied by the $a_{i t}$ coordinates (the vectors) and act as stretching or shrinking factors for the vectors.

As mentioned, these reparameterizations can aid in the interpretation of the derived dimensions (cf. Bentler \& Weeks, 1978; Bloxom, 1978; de Leeuw \& Heiser, 1980; Noma \& Johnson, 1977), and can replace the post-analysis property-fitting methods often used to aftempt to interpret results. In addition, as shall be discussed, the imposition of these sets of reparameterizations can provide an effective tool for understanding the nature of the latent construct under investigation.

It should be noted that when a linear function replaces a product or subject coordinate, the number of background variables in the linear function cannot exceed the number of entities that exists for those variables. For example, if $J$ products have $L$ attributes, $J \geq L$, since, at most, only $J T$ coordinates can be identified (excluding rotational indeterminacy). Similarly, if $I$ subjects have $N$ background variables, $I \geq N$, since, at most, only $I T$ coordinates can be identified (excluding rotational indeterminacy). Thus, in most applications, such reparameterizations actually improve the degrees of freedom of the model by reducing the number of parameters to be estimated. In all cases, the degrees of freedom of any of the models are equal to or greater than those in competing joint space models, as shown below.

\section{Degrees of Freedom}

Researchers typically collect $I\{[J(J-1)] / 2\}$ independent paired comparison responses in an application. Defining the degrees of freedom of the model as the effective number of independent parameters, the degrees of freedom can easily be calculated for the various models accommodated by this method. These are shown in Table 1, where it is assumed there is interest in estimating $\tau_{i}$ where appropriate. Note that in all models an adjustment of $T^{2}$ is required due to the well-known invariance of such bilinear models to nonsingular transformations (Kruskal, 1978). Also, in models where $b_{j t}$ is not reparameterized, a constant vector $\mathbf{c}$ can be added to all stimulus vectors $b_{j}$ and not affect the choice probabilities in Equation 3. This necessitates a subtraction of $T$ from the degrees of freedom. Finally, in estimating $\tau_{i}$, the overall scale can be set by fixing one $\tau_{i}=1$. 
Volume 10 Number 1 March 1986
84 APPLIED PSYCHOLOGICAL MEASUREMENT

Table 1

Degrees of Freedom Calculations for the Various Models

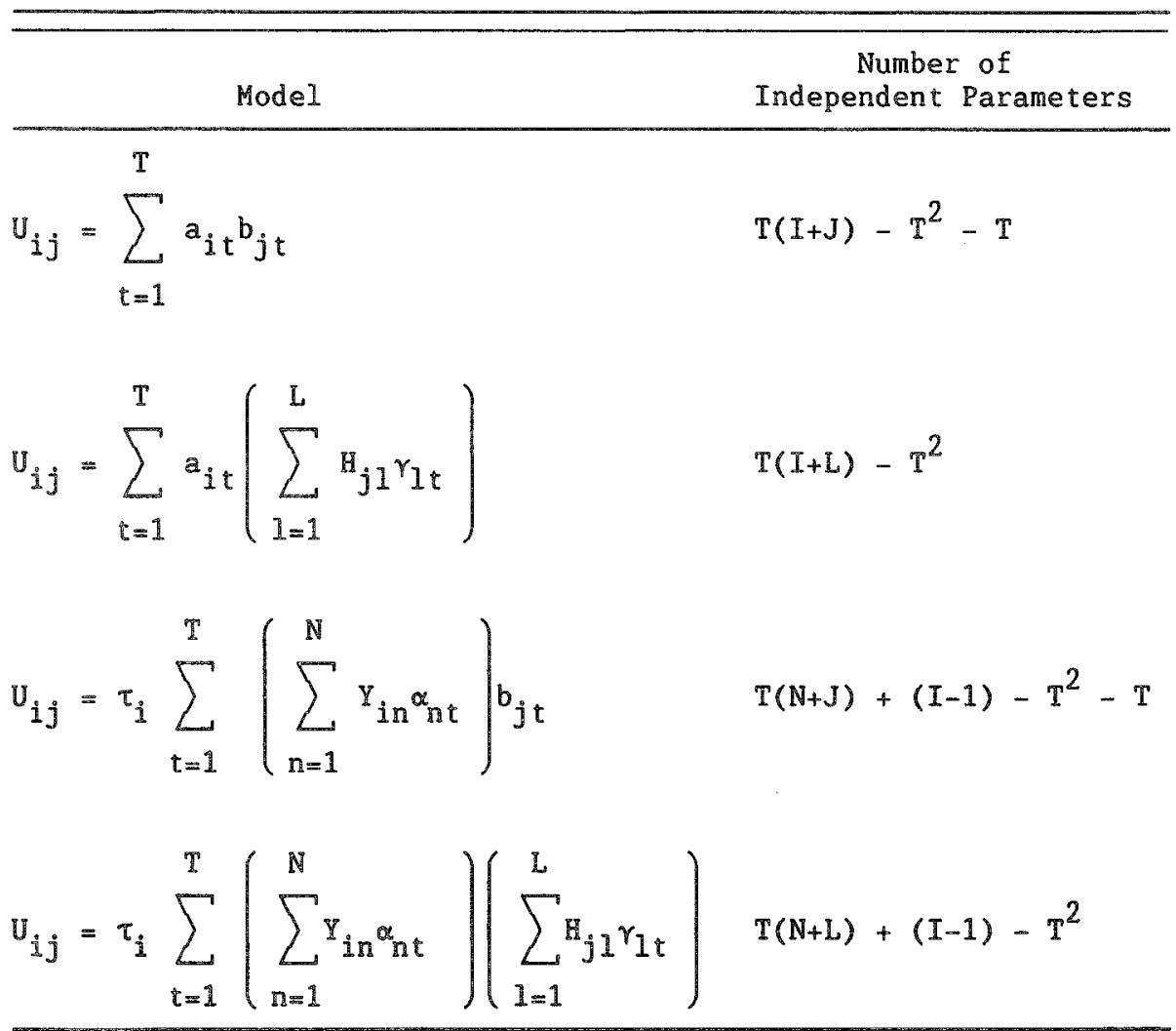

\section{The Algorithm}

Maximum likelihood methods are used to estimate the desired set of parameters to maximize $\ln L$ (or minimize $-\ln L$ ) in Equation 7. The method of conjugate gradients (Fletcher \& Reeves, 1964) is used to solve this nonlinear, unconstrained optimization problem. The partial derivatives of $\ln L$ in Equation 7 with respect to the various parameters are:

$$
\begin{aligned}
\frac{\partial \ln L}{\partial a_{i t}} & =\sum_{j<k}^{J} \frac{\delta_{i j k}-\Phi(\cdot)}{\Phi(\cdot)[1-\Phi(\cdot)]} \phi(\cdot)\left(b_{j t}-b_{k t}\right), \\
\frac{\partial \ln L}{\partial b_{j t}} & =\sum_{i=1}^{I} \sum_{k>j}^{J} \frac{\delta_{i j k}-\Phi(\cdot)}{\Phi(\cdot)[1-\Phi(\cdot)]} \phi(\cdot) \tau_{i} a_{i t} \\
\frac{\partial \ln L}{\partial \gamma_{l t}} & =\sum_{i=1}^{l} \sum_{j<k}^{J} \frac{\delta_{i j k}-\Phi(\cdot)}{\Phi(\cdot)[1-\Phi(\cdot)]} \phi(\cdot) \tau_{i} a_{i t}\left(H_{j l}-H_{k l}\right), \\
\frac{\partial \ln L}{\partial \alpha_{n t}} & =\sum_{i=1}^{I} \sum_{j<k}^{J} \frac{\delta_{i j k}-\Phi(\cdot)}{\Phi(\cdot)[1-\Phi(\cdot)]} \phi(\cdot) \tau_{i} Y_{i n}\left(b_{j t}-b_{k t}\right)
\end{aligned}
$$


$\frac{\partial \ln L}{\partial \tau_{i}}=\sum_{j<k}^{J} \frac{\delta_{i j k}-\Phi(\cdot)}{\Phi(\cdot)[1-\Phi(\cdot)]} \phi(\cdot) \sum_{i=1}^{T} a_{i t}\left(b_{j t}-b_{k t}\right)$

where $\phi(\cdot)$ represents the evaluation of the standard normal density at $(\cdot)$. For sake of convenience, assume that the relevant parameters to be estimated are contained in the vector $\theta$ and that $\nabla \mathbb{Z}$ is the vector of partial derivatives for this set of parameters.

The complete conjugate gradient procedure can be summarized (cf. Rao, 1979) as follows:

1. Start with initial parameter estimates $\theta^{(1)}$; set the iteration counter (MIT) $=1$.

2. Set the first search direction $\mathbf{S}^{(1)}=-\nabla \mathbb{Z}^{(1)}$.

3. Find $\theta^{(2)}$ according to the relation:

$\theta^{(2)}=\theta^{(1)}+u^{(1)} \mathbf{S}^{(1)}$,

where $\boldsymbol{u}^{(1)}$ is the optimal step length in the direction $\mathbf{S}^{(1)}$. The optimal step size is found by quadratic interpolation methods. Set MIT $=2$.

4. Calculate $\nabla Z^{(2)}$ and set

$$
\mathbf{S}^{(\mathrm{MIT})}=-\nabla \mathbf{Z}^{(\mathrm{MIT})}+\frac{\left[\nabla Z^{(\mathrm{MIT})}\right]^{\prime}\left[\nabla \mathbb{Z}^{(\mathrm{MTT})}\right]}{\left[\nabla \mathbf{Z}^{(\mathrm{MIT}-1)}\right]^{\prime}\left[\nabla Z^{(\mathrm{MIT}-1)}\right]} \mathbf{S}^{(\mathrm{MIT})}
$$

5. Compute the optimal step length $u^{(\mathrm{Mrr})}$ in the direction $\mathbf{S}^{(\mathrm{MIT})}$, and find

$$
\boldsymbol{\theta}^{(\mathrm{MIT}+1)}=\boldsymbol{\theta}^{(\mathrm{MIT})}+u^{(\mathrm{MIT})} \mathbf{S}^{(\mathrm{MIT})} .
$$

6. If $\theta^{(M T T+1)}$ is optimal, stop. Otherwise, set MIT $=$ MIT +1 and go to step 4 above (i.e., undertake another iteration).

It has been demonstrated empirically that conjugate gradient procedures can avoid the typical "cycling' often encountered with steepest descent algorithms. In addition, they demonstrate valuable quadratic termination properties (Himmelblau, 1972) - that is, conjugate gradient procedures will typically find the globally optimum solution for a quadratic function in $n$ steps, where $n$ is the number of parameters to be solved. Note that since $\ln L$ in Equation 7 has an upper bound of zero, and since each estimating stage (or iteration) of the likelihood maximization can be shown to increase $\ln L$, a limiting sums argument can be used (Courant, 1965) to prove convergence to at least a locally optimum solution.

This conjugate gradient method is particularly useful for optimizing functions of several parameters since it does not require the storage of any matrices (as is necessary in quasi-Newton and second derivative methods). However, as noted by Powell (1977), the rate of convergence of the algorithm is linear only if the iterative procedure is "restarted" accasionally. Restarts have been implemented in the algorithm automatically depending on successive improvement in the objective function.

A number of goodness-of-fit measures are computed for this model:

1. The $\ln$ likelihood function: $\ln L$,

2. A deviance measure (McCullagh \& Nelder, 1983; Nelder \& Wedderbrun, 1972):

$$
D=-2\left[\sum_{i=1}^{I} \sum_{j<k}^{J} \delta_{i j k} \ln \left(\hat{p}_{i j k}\right)+\left(1-\delta_{i j k}\right) \ln \left(1-\hat{p}_{i j k}\right)\right]=-2 \ln L
$$

where $\hat{p}_{i j k}$ is the estimated probability that subject $i$ finds stimulus $j$ more satisfying than stimulus $k$ as expressed in Equation 3. Note that nested models can be tested as the difference between respective deviance measures. This difference is (asymptotically) $\chi^{2}$ distributed with the difference in model degrees of freedom, providing the appropriate $\chi^{2}$ test degrees of freedom. This test is appropriate in testing dimensionality as well as the various models described in Table 1 because of the nested terms. Recall, however, that this is an asymptotic test. One obvious problem with this approach concerns 
incidental parameters in the likelihood function (i.e., parameters whose order varies according to the order of $\Delta$, such as the $a_{i t} \mathrm{~s}$ ). According to Anderson (1980), maximum likelihood estimators in such cases may not be consistent. This is particularly relevant in the present case since there are no replications, thus presenting an avenue of needed research for the proposed procedure if the asymptotic test is to be used for hypothesis testing. However, the simple monte carlo analysis provided in the next section renders some insight concerning the value of the test statistic.

3. The proportion of correct predictions in $\mathbf{\Delta}$. Here, the proportion of times the solution correctly predicts $\delta_{i j k}$ is calculated for the total sample as well as for each subject.

\section{Symthetic Data Results}

Table 2 presents values of $\mathbf{A}=\left\|a_{i t}\right\|$ and $B=\left\|b_{j l}\right\|$ for $I=J=4$ and $T=2$, for the nonreparameterized model. The configurations were constructed so that $\mathbf{A}=\mathbf{B}$ and so that each subject/stimulus was in each quadrant of a two-dimensional space. From this configuration for the model with no reparameterizations, $\Delta=\left\|\delta_{i j k}\right\|$ was created, with error from a standard normal distribution. Table 2 presents the synthetic $\Delta$ array of resulting paired comparisons data where a " 1 " indicates that the row stimulus dominates the column stimulus for that subject. The value of the likelihood function was -2.772 for these parameters.

The analysis of $\Delta$ was conducted in $T=1$ and $T=2$ dimensions using the nonreparameterized model to estimate $\mathbf{A}$ and $\mathbf{B}$. Table 3 presents the results of the one- and two-dimensional solutions. Note that the two-dimensional solution is significantly better than the one-dimensional solution. As can be seen from Table 3, even if the appropriateness of the asymptotic $\chi^{2}$ test in this model were seriously questioned,

Table 2

Synthetic Data for Monte Carlo Analysis

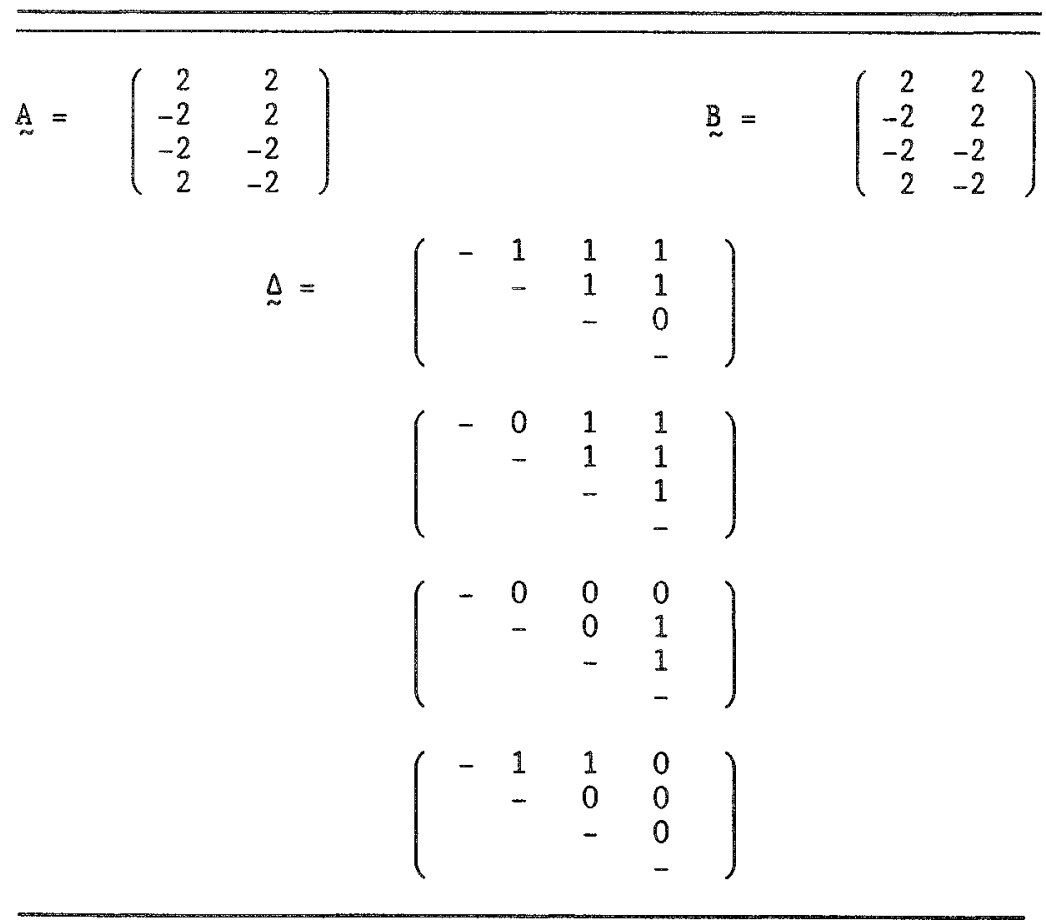


Table 3

Monte Carlo Results

\begin{tabular}{|c|c|c|c|c|c|}
\hline Dimension & $\begin{array}{l}\text { Iterations } \\
\text { Required for } \\
\text { Convergence }\end{array}$ & In $L$ & D & $\begin{array}{l}\text { Difference } \\
\text { of Deviance } \\
\text { Measures }\end{array}$ & $\begin{array}{r}\text { Proportion } \\
\text { of Correct } \\
\text { Predictions }\end{array}$ \\
\hline $\begin{array}{l}T=1 \\
T=2\end{array}$ & $\begin{array}{l}28 \\
25\end{array}$ & $\begin{array}{r}-10.150 \\
-0.013\end{array}$ & $\begin{array}{r}20.300 \\
0.026\end{array}$ & $20.274 x$ & $\begin{array}{r}.750 \\
1.000\end{array}$ \\
\hline
\end{tabular}

$\star_{\mathrm{p}} \leq .01$

the two-dimensional solution renders better predictive accuracy than the one-dimensional solution by $25 \%$. The derived two-dimensional solution also provides a higher $\ln L$ value than the original parameters in Table 2.

Figure 1 depicts the resulting two-dimensional solution drawn for the four hypothetical subjects and four hypothetical stimuli. Note the correspondence between Subject 1 and Stimulus A, Subject 2 and Stimulus B, Subject 3 and Stimulus C, and Subject 4 and Stimulus D, as was denoted by the original $\mathbf{A}$ and $\mathbf{B}$ configuration in Table 2 . From Figure 1, it is also easy to visualize what stimulus dominates which other stimulus by projecting the stimuli onto a subject's vector and examining the ordering on that vector. Table 4 presents these "latent projection scores" ( $U_{i j}$ as defined in Equation 1) of the four hypothetical stimuli for the four hypothetical subjects as predicted from the solution. Note how the main diagonal elements of this matrix $\mathbf{U}=\mathbf{A B}^{\prime}$ dominate each of the corresponding row entries.

\section{Figure 1}

Two-Dimensional Solution for Synthetic Data

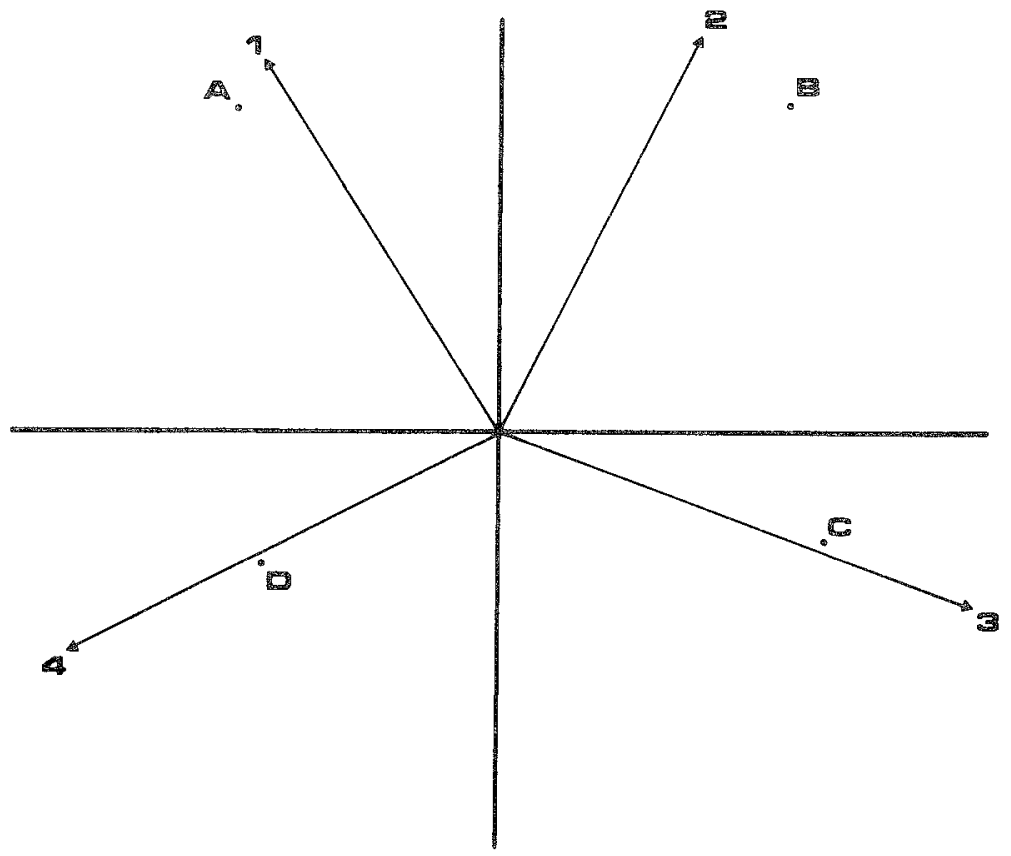


Table 4

Latent Projection Scores

\begin{tabular}{crrrr}
\hline & \multicolumn{4}{c}{ Stimulus } \\
\cline { 2 - 5 } Subject & $\mathrm{A}$ & $\mathrm{B}$ & $\mathrm{C}$ & $\mathrm{D}$ \\
\hline 1 & 8.64 & 4.11 & -4.70 & -0.53 \\
2 & 5.04 & 8.80 & 0.06 & -4.32 \\
3 & -7.39 & 1.51 & 6.22 & -2.70 \\
4 & 0.27 & -8.35 & -3.86 & 5.30 \\
\hline
\end{tabular}

Although the results of this one synthetic data set are promising, it is clear that more testing is required on other data sets using a comprehensive monte carlo analysis. For example, it would be desirable to examine the performance of the algorithm as a number of data, model, and error factors are systematically manipulated by way of, say, an experimental design. Positive results for this one small data set do not imply that this procedure will recover known structure in all monte carlo scenarios.

\section{An Application in Consumer Satisfaction Measurement}

\section{Major Constructs in the Consumer Satisfaction Literature}

Despite the fact that references to consumer satisfaction are legion, the literature on consumer satisfaction research reveals that less is known about the psychological determinants of this concept than might be expected. If analysis is constrained to those concepts thought to be causally antecedent to an expression of satisfaction, five such determinants emerge. The following discussion is restricted to the most general case of the process of satisfaction formation, thought to be common to all consumers. Excluded are individual-specific factors such as demographics (Liefeld, Edgecombe, \& Wolfe, 1975; Warland, Herrmann, \& Willits, 1975), personality (Ware, 1978), and optimistic/pessimistic tendencies (Westbrook, 1980).

Also excluded from this example is the effect of product performance, the first and most obvious of the major determinants. This was done for two reasons. First, the effect of product attribute performance on satisfaction judgments is axiomatic and well documented in numerous literatures, including job satisfaction (Brief \& Aldag, 1975; Hackman \& Oldham, 1975), patient satisfaction (Pope, 1978; Ware \& Snyder, 1975), life in general (Andrews \& Withey, 1976; Campbell, Converse, \& Rodgers, 1976), and products and services (e.g., Westbrook, 1981), despite the fact that it cannot explain all of the variance in satisfaction for methodological and theoretical reasons (Scapello \& Campbell, 1983). Second, performance can be viewed as having indirect effects through other more immediate determinants, particularly expectancy disconfirmation.

Expectancy disconfirmation. Expectancy disconfirmation is actually two processes consisting of the formation of expectations and the disconfirmation of those expectations through performance comparisons. As presented by Oliver (1980) in the marketing literature, consumers are believed to form expectations of product performance characteristics prior to purchase. Subsequent purchase and usage reveals actual performance levels, which are then compared to expectation levels using a better-than, worse-than heuristic. The resulting judgment is labeled as negative disconfirmation if the product is worse than expected, positive disconfirmation if better than expected, and simple confirmation if as expected.

Although linked on a definitional basis, the two components of the paradigm, expectation and 
disconfirmation, have been shown to have independent effects akin to adaptation level predictions (Helson, 1965). The expectation level appears to provide a baseline around which disconfirmation judgments are made; the higher (lower) a person's expectations, the higher (lower) the subsequent satisfaction judgment, all elements being equal. The disconfirmation effect is also straightforward. Positive disconfirmation is thought to enhance satisfaction, whereas negative disconfirmation is posited to decrease it. Confirmation simply maintains the adaptation level. Support for this paradigm is rather consistent (Bearden \& Teel, 1983; LaBarbera \& Mazursky, 1983; Oliver, 1977, 1980; Swan \& Trawick, 1981) with mixed results apparent only when the confounding influence of performance is introduced (Churchill \& Surprenant, 1982).

Attribution. The attribution paradigm used here is due to the work of Weiner, Frieze, Kukla, Reed, Rest, and Rosenbaum (1971) who proposed that outcomes that can be construed as successes or failures (e.g., good and poor purchases) elicit causality inferences along the two dimensions of locus of control (when the outcome was either under volitional control or the result of an external agent) and stability (variability) of the cause. Volitional outcomes (internal locus) are attributed either to ability (a stable trait) or effort (a variable activity). Outcomes resulting from nonvolitional influences are attributed to many factors, some of which are invariant (e.g., task difficulty), others of which are unstable (e.g., "luck"). Weiner, Russell, and Lerman (1978) later broadened this scheme to include the kinds of emotions that result as a factor of specific attributions.

Limiting discussion to the locus of control concept in the domain of successes (the context investigated here), Weiner et al. (1978, pp. 71-77) showed that satisfaction scores were higher for ability and effort attributions than for attributions to the task, others' efforts, or "luck". Moreover, satisfaction was found to describe internal locus attributions more frequently; external attribution responses tended to include appreciation and gratification. Interestingly, this scheme is largely unresearched in the domain of consumer satisfaction.

Equity. Although equity in exchange has proven to be of continued interest in the organizational behavior field (e.g., Cosier \& Dalton, 1983), less attention has been evident in the marketing literature, with the notable exception of Huppertz, Arenson, and Evans (1978). Generally, the basic equity "formula" suggests that parties to an exchange will feel equitably treated and thus, "satisfied," if the ratio of their outcomes to inputs is identical. While this is difficult to show mathematically (Harris, 1983), the proposition that persons in an exchange form judgments about inputs and outcomes for themselves and their exchange partners is not in dispute.

In this scheme, satisfaction is thought to exist when the focal person (i.e., the consumer in the present case) perceives either: (1) that the outcome to input ratios are equal - the "fairness" dimension, or (2) that he/she has bettered the other in the transaction-the "preference" dimension. Although the literature is unclear as to which equity dimension can be expected to dominate in any given situation (Messick \& Sentis, 1983), the focus in the present context is on the preference dimension, which predicts that a person's satisfaction increases as his/her outcomes increase relative to those of the other party (positive inequity), and decrease as a person's outcomes decrease relative to those of the other (negative inequity). This proposition assumes that inputs are held constant or are unknown to both parties.

The preceding review has suggested four determinants of satisfaction responses. Excluding performance for reasons noted earlier, these are (1) the expectation level, (2) disconfirmation, (3) attribution, and (4) inequity. The respective theories suggest satisfaction levels should be higher (lower) for high (low) expectations, positive (negative) disconfirmation, internal (external) attributions, and positive (negative) inequity. While individual studies have examined each of these influences separately and, in some cases, in the presence of one other influence, no study has examined all in tandem. Thus, the relative effects of each are not known. 


\section{Research Vehicle}

To provide a realistic perspective for the application presented here, a quasi-purchase situation was needed that implicitly contained all of the elements required for operation of the major satisfaction constructs discussed above. This necessitated that the product situation involve: (1) the formation of expectations as integral rather than incidental to the purchase, (2) the ability to disconfirm objectively those expectations, (3) the likelihood that an agent other than the consumer could make the product selection, and (4) the ability for the buyer to determine precisely the outcomes of all parties to the transaction. The first two conditions provide for the operation of expectancy disconfirmation, while the third and fourth allow for locus attributions and inequity mechanisms.

This goal was accomplished by way of simulated stock market trading scenarios. A stock market transaction was selected because it contained all elements noted above. Expectations are implicit in this consumption decision because no rational investor would risk funds in the market unless a gain were expected. Disconfirmation is present because the exact return is known at any instant of time and is easily compared to expectations. Locus of control attributions are possible because investors commonly make their own decisions (internal locus) in some instances, or rely exclusively on the advice of a broker, a brokerage, or other professional recommendations (external locus) in others. Finally, since individual investors almost exclusively use an agent (i.e., a broker) for stock transactions, the outcomes of both parties are easily compared. Whereas the investor realizes a gain or loss net of commissions, the broker gains the commission which is reported on the resulting transaction acknowledgment.

With this justification, eight stock market transaction scenarios were designed from a fractional factorial design (i.e., an orthogonal array) of expectancy, disconfirmation, attribution, and inequity possibilities. The eight profiles allow for main effects estimation only. Because the scenarios were to be judged in paired comparison presentation formats, the 28 pairings resulting from the eight scenarios were the maximum number that could be accommodated without respondent fatigue. As a result, more complex designs allowing for the estimation of higher-order interaction terms were not used since they would require substantially more experimental profiles and thus more judgments. Several marketing studies have documented the robust use of such main effects designs for conjoint analysis (cf. Green \& Srinivasan, 1978) even in the presence of some higher-order interaction terms. The selected design allowed for 3 two-level factors and 1 three-level factor without violating the orthogonality restrictions (Addelman, 1962), and is presented in Table 5.

Description of the design matrix follows. The constructs are elaborated in order of their occurrence in an actual stock market trade.

1. Attribution. The stock transaction described in the scenario was said to have been the result of the investor's research and decision (internal attribution) or of the broker's research and recommendation (external attribution).

Table 5

Design Matrix

\begin{tabular}{ccccc}
\hline Scenario & Attribution & Expectation & Disconfirmation & Inequity \\
\hline 1 & External & Low & None & Negative \\
2 & Internal & High & None & Positive \\
3 & External & Low & Positive & Positive \\
4 & Internal & High & Positive & Negative \\
5 & External & High & Negative & Negative \\
6 & Internal & Low & Negative & Positive \\
7 & External & High & Positive & Positive \\
8 & Internal & Low & Positive & Negative \\
\hline
\end{tabular}


2. Expectation. The research leading up to the decision, whether it be the investor's or the broker's, was described as predicting that the stock would either better the market (e.g., Standard and Poor's 500 ) by $5 \%$ in six months (high expectations) or would simply pace the market (low expectations) in the same time frame.

3. Disconfirmation. The stock's performance was described as either (1) bettering a person's expectations by $5 \%$ for positive disconfirmation (10\% above the market's percentage performance in the $5 \%$ expectation case, 5\% above for "market" expectations), (2) matching expectations in the no disconfirmation case (simple confirmation), or (3) falling short of expectations by $5 \%$ in the negative disconfirmation case. Because there were three investigated levels of disconfirmation (positive, zero, and negative), the separate effects of positive and negative disconfirmation could be tested separately against zero disconfirmation in the design. Thus, two main effects were estimated for the disconfirmation construct.

4. Inequity. For positive inequity, the commission structure was defined so that the investor's performance (i.e., the actual monetary outcome net of commissions) was $20 \%$ above the broker's twoway (purchase, sale) commission, whereas for negative inequity, the broker's commission exceeded the investor's gain by $20 \%$.

An actual scenario (profile 4) is shown in Table 6. All numbers reflecting market performance, stock performance, and commissions are well within historical norms for short-term (six-month) performance periods. Brokerage commissions reflect those representative of full-service brokers on a two-way transaction for a $\$ 50$ stock. The $3^{1} 2^{3}$ design matrix in Table 5 was transformed into a dummy variable matrix with an intercept term for incorporation into the analyses that follow. The 1 three-level factor was needed to accommodate the disconfirmation effect as noted.

\section{Procedire}

Business school students at the University of Pennsylvania with experience in the financial markets were recruited to participate in a study of responses to stock market transaction outcomes. As an incentive, $\$ 5.00$ was offered; 30 subjects were recruited, including 20 males and 10 females. Subjects were presented with all 28 pairs of the eight scenarios in random order. Each scenario was alternated with the remaining seven so that it appeared in the first and second positions an equal number of times. After reading and comparing the two scenarios, subjects were asked to state which one was "most satisfying to you

Table 6

Sample Scenario With Internal Attribution Locus, High Expectations, Positive Disconfirmation, and Negative Inequity

1. Based on your personal research, you feel strongly about the prospect for Stock X.

2. This research suggests that the stock should outperform the S\&P 500 by $5 \%$ on a sixs month basis.

3. You buy 100 shares at $\$ 50$ a share through your local broker.

4. Shortly after the six month long-term holding period, you decide to sell you shares to "lock in the gain."

5. Over your holding period, the market was up $1 \%$ while Stock $\mathrm{X}$ rose to $\$ 55.50(+11 \%)$, thus outperforming the market on a percentage basis and exceeding your expectations.

6. You net a $\$ 250$ profit after the gross proceeds (\$550) are adjusted for $\$ 300$ in broker's commissions on the purchase and sale of the stock. 
personally." Because of the difficulty involved in assessing the large number of experimental profiles, it was thought that the paired comparison judgment approach would be the easiest and most reliable method as opposed to a continuous rating or ranking task.

\section{Analysis}

To determine how well, if at all, the latent satisfaction construct could be defined in terms of the hypothesized manifest variables, an analysis of the $30 \times 8 \times 8 \Delta$ array was conducted in $T=1,2$, and 3 dimensions with the probabilistic vector model, using the reparameterization option for the $b_{j t} \mathrm{~s}$ as a function of the design matrix presented in Table 5. Random starting configurations were generated for each analysis. Reparameterization options for $a_{i t}$ were not used since the students were fairly homogeneous with respect to demographics (except for gender).

Table 7 presents the results for the three analyses. If it is assumed that the asymptotic $\chi^{2}$ test is valid, it is apparent that the $T=2$ dimensional solution is the most appropriate for the present data. Even if such a test were ignored, the differences in the proportion of correct predictions between the three solutions clearly indicate that the $T=2$ dimensional solution is the most parsimonious one for this application. (Note that another two-dimensional solution extracted from a different random start produced nearly identical results as measured by canonical correlations between the different $\mathbf{A}, \mathbf{B}$, and $\boldsymbol{\gamma}$ solutions.)

Table 8 presents the correlations between each design variable and the $\mathbf{B}$ coordinates. The first dimension is clearly dominated by attribution, accounting for nearly two-thirds of the variance on that dimension. The second dimension is dominated by the two disconfirmation states with their large correlations having the expected opposite signs. (These correlations will vary according to the type of rotation utilized for interpreting $\mathbf{A}$ and/or $\mathbf{B}$.)

Figure 2 presents the two-dimensional solution where the $\gamma_{l t}$ and $a_{i t}$ are both represented as vectors (normalized to equal length, for convenience) and the profiles as points (numbers). The letters $\mathrm{A}$ through $F$ represent the $\gamma_{t t}$ coordinates for the columns/variables of the design matrix, where

$A=$ the location of $\gamma_{1}$ for the intercept term representing the effects of all

design variables at their coded level of zero,

$B=$ the location of $\gamma_{2}$ representing the impact of internal (self) attribution,

$\mathrm{C}=$ the location of $\gamma_{3}$ representing the impact of high expectations,

$\mathrm{D}=$ the location of $\boldsymbol{\gamma}_{4}$ representing the impact of positive disconfirmation,

$\mathrm{E}=$ the location of $\gamma_{5}$ representing the impact of negative disconfirmation, and

$F=$ the location of $\gamma_{6}$ representing the impact of positive inequity.

These $\gamma_{1}$ vectors can aid in interpreting the resulting dimensions extracted in the analysis. Specifically, if the "independent" variables in $\mathbf{H}$ are orthonormal and the $\mathbf{B}$ coordinates are centered by dimension,

Table 7

Consumer Satisfaction Analyses

\begin{tabular}{|c|c|c|c|c|c|}
\hline Dimension & $\begin{array}{l}\text { Iterations } \\
\text { Required for } \\
\text { Convergence }\end{array}$ & $\operatorname{In} L$ & D & $\begin{array}{l}\text { Difference } \\
\text { of Deviance } \\
\text { Measures }\end{array}$ & $\begin{array}{l}\text { Proportion } \\
\text { of Correct } \\
\text { Predictions }\end{array}$ \\
\hline $\begin{array}{l}\mathrm{T}=1 \\
\mathrm{~T}=2 \\
\mathrm{~T}=3\end{array}$ & $\begin{array}{l}22 \\
31 \\
36\end{array}$ & $\begin{array}{l}-581.006 \\
-346.630 \\
-327.951\end{array}$ & $\begin{array}{r}1162.012 \\
693.260 \\
655.902\end{array}$ & $\begin{array}{l}468.752 * \\
37.358\end{array}$ & $\begin{array}{l}.515 \\
.804 \\
.831\end{array}$ \\
\hline
\end{tabular}

$* \mathrm{p} \leq .01$ 
Table 8

Correlations Between Design Variables $(H)$ and Derived Stimulus Coordinates $(\underset{\sim}{B})$

\begin{tabular}{lcr}
\hline & \multicolumn{3}{c}{ Dimension } \\
\cline { 3 - 3 } Design Variable & $\mathrm{I}$ & II \\
\hline Attribution & .814 & .240 \\
Expectancy & .146 & .343 \\
Positive Disconfirmation & .471 & .847 \\
Negative Disconfirmation & -.506 & -.756 \\
Inequity & -.111 & -.034 \\
\hline
\end{tabular}

the cosine of the angle between a $\gamma$ vector and a coordinate axis is equal to the correlation between that th feature or attribute variable in $\mathbf{H}$ and the corresponding dimension. Unfortunately, this interpretation is not appropriate in Figure 2 given that $\mathbf{H}$ is not orthonormal.

Figure 2 aptly shows that the majority of subjects find profiles with self (internal) attribution, high expectations, and positive disconfirmation most satisfying, though there are considerable individual differences. This finding conforms to earlier hypotheses and to what is predicted from the literature. In fact, each subject's own satisfaction scale for these eight profiles can be approximated by computing $\mathbf{U}_{i}=$ $\mathbf{A B}^{\prime}$, which measures the projections of the profile points $(\mathbf{B})$ onto the subject vectors $\left(\mathbf{A}_{i}\right)$. Table 9 presents the latent satisfaction scores for all 30 subjects. These derived latent scores are interval scales and are conditional in that the magnitudes of the numbers cannot really be compared for different subjects. However, by surveying Table 9 , it is readily apparent that Profile 4 is the most satisfying for 25 out of the 30 subjects. This profile, shown in detail in Table 6, is composed of internal attribution, high expectations, positive disconfirmation, and negative inequity. Note that other popular profiles, outlined

Figure 2

Two-Dimensional Solution for Consumer Satisfaction Data

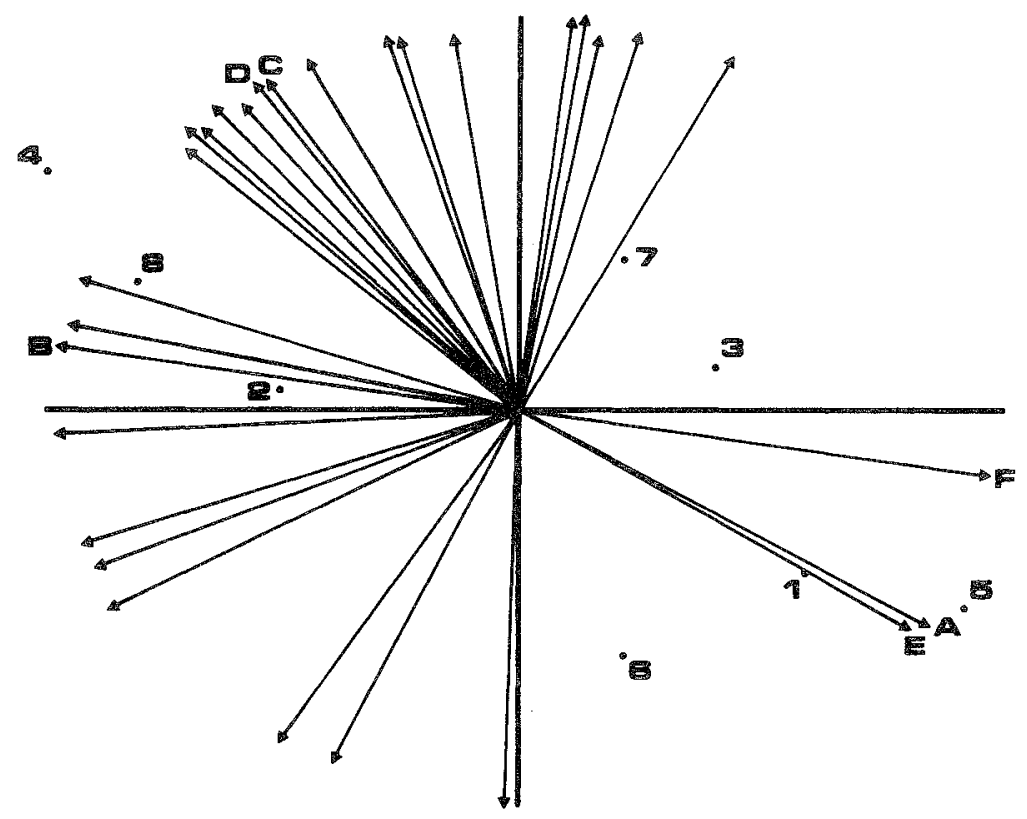


Table 9

Derived Latent Satisfaction Scores for the Eight Profiles by Subject

\begin{tabular}{|c|c|c|c|c|c|c|c|c|}
\hline \multirow[b]{2}{*}{ Subject } & \multicolumn{8}{|c|}{ Profile } \\
\hline & 1 & 2 & 3 & 4 & 5 & 6 & 7 & 8 \\
\hline 1 & 0.023 & 0.174 & -0.242 & -0.005 & -0.053 & 0.350 & -0.368 & 0.121 \\
\hline 2 & 0.161 & 0.197 & -0.339 & -0.213 & 0.099 & 0.645 & -0.593 & 0.042 \\
\hline 3 & -1.264 & 0.706 & -0.330 & 2.065 & -1.808 & -1.104 & 0.303 & 1.433 \\
\hline 4 & -0.587 & 0.665 & -0.601 & 1.019 & -0.994 & 0.081 & -0.512 & 0.930 \\
\hline 5 & -0.999 & -0.040 & 0.532 & 1.527 & -1.156 & -1.923 & 1.395 & 0.664 \\
\hline 6 & -1.747 & 0.979 & -0.461 & 2.855 & -2.501 & -1.520 & 0.412 & 1.983 \\
\hline 7 & -0.265 & -0.072 & 0.223 & 0.394 & -0.279 & -0.619 & 0.490 & 0.128 \\
\hline 8 & -0.092 & -0.145 & 0.237 & 0.116 & -0.042 & -0.426 & 0.402 & -0.050 \\
\hline 9 & -0.979 & 1.209 & -1.135 & 1.715 & -1.703 & 0.312 & -1.048 & 1.629 \\
\hline 10 & -0.287 & 0.018 & 0.114 & 0.444 & -0.346 & -0.501 & 0.344 & 0.214 \\
\hline 11 & 0.292 & -0.041 & -0.085 & -0.456 & 0.362 & 0.470 & -0.306 & -0.236 \\
\hline 12 & -1.171 & 1.025 & -0.798 & 1.979 & -1.845 & -0.370 & -0.437 & 1.618 \\
\hline 13 & -0.786 & 0.246 & 0.051 & 1.250 & -1.036 & -1.025 & 0.561 & 0.739 \\
\hline 14 & -1.127 & 0.657 & -0.331 & 1.846 & -1.625 & -0.936 & 0.217 & 1.299 \\
\hline 15 & -1.376 & 0.806 & -0.409 & 2.254 & -1.985 & -1.135 & 0.257 & 1.588 \\
\hline 16 & -1.410 & 1.077 & -0.752 & 2.354 & -2.149 & -0.721 & -0.223 & 1.825 \\
\hline 17 & -1.874 & 1.107 & -0.570 & 3.071 & -2.708 & -1.528 & 0.330 & 2.171 \\
\hline 18 & -1.770 & 1.527 & -1.177 & 2.986 & -2.778 & -0.597 & -0.619 & 2.428 \\
\hline 19 & -3.069 & 1.751 & -0.852 & 5.020 & -4.407 & -2.612 & 0.661 & 3.507 \\
\hline 20 & -2.067 & 1.119 & -0.493 & 3.370 & -2.940 & -1.866 & 0.563 & 2.314 \\
\hline 21 & -0.929 & 0.360 & -0.032 & 1.489 & -1.256 & -1.090 & 0.530 & 0.928 \\
\hline 22 & -1.258 & 0.034 & 0.558 & 1.937 & -1.494 & -2.273 & 1.593 & 0.902 \\
\hline 23 & -1.548 & 1.336 & -1.031 & 2.611 & -2.429 & -0.520 & -0.543 & 2.124 \\
\hline 24 & -1.096 & 0.793 & -0.526 & 1.822 & -1.650 & -0.638 & -0.088 & 1.384 \\
\hline 25 & -2.098 & 0.801 & -0.056 & 3.362 & -2.831 & -2.482 & 1.218 & 2.087 \\
\hline 26 & -1.401 & 0.688 & -0.214 & 2.268 & -1.951 & -1.423 & 0.556 & 1.498 \\
\hline 27 & -0.872 & 0.930 & -0.816 & 1.502 & -1.450 & 0.019 & -0.649 & 1.335 \\
\hline 28 & -0.808 & 0.721 & -0.569 & 1.367 & -1.279 & -0.230 & -0.329 & 1.127 \\
\hline 29 & -0.733 & 0.842 & -0.766 & 1.273 & -1.246 & 0.122 & -0.633 & 1.170 \\
\hline 30 & -2.141 & 1.232 & -0.607 & 3.503 & -3.078 & -1.804 & 0.442 & 2.454 \\
\hline
\end{tabular}

in Table 5, were Profiles 2 and 8, which contained internal attribution and either positive or no disconfirmation. It is interesting to note that Profiles 3, 6, and 7 are not as popular since they have either internal attribution or positive disconfirmation, but not both. Profiles 1 and 5 are clearly "dissatisfying" in that they contain both external attribution and negative disconfirmation.

Finally, Table 10 presents the proportion of correct predictions for each subject in the study. This analysis is valuable in identifying any outliers in the sample. Note that, in all cases, the probabilistic vector model does better than a random selection approach, which would generate (on the average) a $50 \%$ accuracy rate. Using this criterion, it is observed that Subjects 11 and possibly 8 are seemingly not all that well fit by this two-dimensional solution.

\section{Discussion}

\section{Summary and Implications for Latent Construct Measurement}

A new MDS methodology for the spatial analysis of paired comparisons data has been presented and contrasted to existing methodologies in terms of model structure, stochastic assumptions, input require- 
ments, and model specification options. The model, its assumptions, and the variety of different reparameterization options available for various analyses have been described. The conjugate gradient algorithm with restarts has been presented with the results from a small synthetic data set. An application of the method to a measurement problem in consumer satisfaction was described in some detail in which four hypothesized determinants were combined using an experimental design matrix. An analysis was performed in which stimulus coordinates (here experimental profiles) were directly reparameterized in terms of a $3^{12} 2^{3}$ design. The procedure produced two dimensions dominated by attribution and disconfirmation, respectively, as well as approximations of each subject's latent satisfaction scale for each of the eight experimental profiles by way of $\mathbf{U}=\mathbf{A B}$.

The usefulness of the technique may best be gauged by the extent to which the determinants of consumer satisfaction were represented on an aggregate and individual basis. Knowing only that the four hypothesized antecedents had been shown to affect satisfaction to varying degrees when tested alone in other contexts, no a priori basis existed for specifying which of the factors would dominate satisfaction judgments or, alternatively, fail to influence satisfaction in the present example. Application of the MDS vector model developed here revealed that internal attribution, a construct attracting little empirical interest

Table 10

Goodness-of-Fit by Subject

\begin{tabular}{|c|c|}
\hline Subject & $\begin{array}{l}\text { Proportion } \\
\text { of Correct } \\
\text { Predictions }\end{array}$ \\
\hline 1 & .750 \\
\hline 2 & .679 \\
\hline 3 & .821 \\
\hline 4 & .714 \\
\hline 5 & .857 \\
\hline 6 & .857 \\
\hline 7 & .714 \\
\hline 8 & .607 \\
\hline 9 & .893 \\
\hline 10 & .679 \\
\hline 11 & .571 \\
\hline 12 & .714 \\
\hline 13 & .750 \\
\hline 14 & .857 \\
\hline 15 & .786 \\
\hline 16 & .857 \\
\hline 17 & .857 \\
\hline 18 & .964 \\
\hline 19 & 1.000 \\
\hline 20 & .929 \\
\hline 21 & .821 \\
\hline 22 & .929 \\
\hline 23 & .893 \\
\hline 24 & .786 \\
\hline 25 & .964 \\
\hline 26 & .893 \\
\hline 27 & .750 \\
\hline 28 & .750 \\
\hline 29 & .857 \\
\hline 30 & .857 \\
\hline
\end{tabular}


in the consumer satisfaction literature, is as important as disconfirmation, a construct for which a fairly adequate volume of support is available. This finding does not underplay the role of disconfirmation, however, as both positive and negative disconfirmation were represented on the first dimension and dominated the second. Rather, the results suggest that factors other than disconfirmation may play a prominent role in certain situations.

As noted, the technique is equally useful at identifying factors thought to underly constructs that have little or no effect in specific contexts. The expectation construct is an example of the first case. Although it demonstrated a modest loading on the second dimension, its effect was small compared to that of disconfirmation. The inequity construct is an example of a null effect, as the findings show virtually no influence on either the first or second dimensions.

Thus, the MDS vector model is capable of illustrating the underlying construct structure of criterion variables that may be difficult to verbalize, are subject to social acceptance effects, or are rife with multicollinearity problems--issues commonly found in the measurement of satisfaction judgments. Moreover, this same versatility should be evident in individual level analyses in that they are subject to the same interpretations as the aggregate analysis. Individual analysis also makes it possible to identify subjects who have no apparent cognitive structure within the confines of the investigated latent construct or determinant set. The implications of analysis of such individuals would appear to be intriguing for the study of information effects.

This methodology should prove equally viable for various other applications when paired comparisons are collected. It can aid in similar measurement problems concerning latent, unobservable constructs such as utility, preference, choice, similarity, risk, or intention/attitude. With the various reparameterization options for $a_{i t}$ and $b_{j t}$, additional flexibility is provided for investigating determinants of both individual differences (e.g., demographic information) and stimulus differences.

Such reparameterization options would also be valuable in utilizing this methodology for an external type of preference MDS analysis generally referred to as conjoint analysis. Here, as in the satisfaction application provided, a design matrix is presented defining object stimulus profiles, and a dominance judgment such as preference or intention to buy is asked in paired comparison form. The methodology then derives the contribution of each object design variable to the resulting derived dimensions. This has proven to be of substantial interest to the marketing profession for product design applications (see DeSarbo et al., 1982).

\section{Future Research}

There are several clear avenues for future investigation concerning this methodology. First, a rigorous monte carlo study should be performed whereby the performance of this new methodology is investigated while a number of data, model, and algorithmic factors are experimentally varied. Second, the small sample properties of the estimators and the $\chi^{2}$ test should be examined in order to justify the asymptotic $\chi^{2}$ test for nested models with incidental parameters and no within-subject replications. Also, further work should proceed in order to extend this methodology to accommodate an unfolding type of model for such paired comparisons data.

\section{References}

Addelman, S. (1962). Orthogonal main-effect plans for asymmetric factorial experiments. Technometrics, 4 , $21-46$.

Anderson, E. B. (1980). Discrete statistical models with social science applications. New York: North Holland.

Andrews, F. M., \& Withey, S. B. (1976). Social indicators of well-being. New York: Plenum Press. 
Bearden, W. O., \& Teel, J. E. (1983). Selected determinants of consumer satisfaction and complaint reports. Journal of Marketing Research, 20, 21-28.

Bechtel, G. G., Tucker, L. R., \& Chang, W. (1971). A scalar product model for the multidimensional scaling of choice. Psychometrika, 36, 369-388.

Bentler, P. M., \& Weeks, D. G. (1978). Restricted multidimensional scaling models. Journal of Mathematical Psychology, 17, 138-151.

Bloxom, B. (1978). Constrained multidimensional scaling in $N$ spaces. Psychometrika, 43, 397--408.

Bock, R. D., \& Jones, L. V. (1968). The measurement and prediction of judgment and choice. San Francisco: Holden-Day.

Box, B. E. P., Hunter, W. G., \& Hunter, J. S. (1978). Statistics for experimenters. New York: Wiley.

Brief, A.P., \& Aldag, R. J. (1975). Employee reactions to job characteristics: A constructive replication. Journal of Applied Psychology, 60, 182-186.

Campbell, A., Converse, P. E., \& Rodgers, W. L. (1976). The quality of American life. New York: Russell Sage Foundation.

Carroll, J. D. (1980). Models and methods for multidimensional analysis of preferential choice (or other dominance) data. In E. D. Lantermann \& H. Feger (Eds.), Similarity and choice (pp. 234-289). Bern: Hans Huber.

Carroll, J. D., Pruzansky, S., \& Kruskal, J. B. (1980). CANDELINC: A general approach to multidimensional analysis of many-way arrays with linear constraints on parameters. Psychometrika, 45, 3-24.

Churchill, G. A., Jr., \& Surprenant, C. (1982). An investigation into the determinants of customer satisfaction. Journal of Marketing Research, 19, 491594.

Cooper, L. G., \& Nakanishi, M. (1983). Two logit models for external analysis of preferences. Psychometrika, 48, 607-620.

DeSarbo, W. S., \& Rao, V. R. (1984). GENFOLD2: A set of models and algorithms for the GENeral UnFOLDing analysis of preference/dominance data. Journal of Classification, 1, 147-186.

De Soete, G., \& Carroll, J. D. (1983). A maximum likelihood method for firting the wandering vector model. Psychometrika, 48, 553-566.

Fechner, G. T. (1860). Elemente der psychophysik. Leipzig: Breitkopf and Haertel.

Fletcher, R., \& Reeves, C. M. (1964). Function minimization by conjugate gradients. Computer Journal, 7, 149-154.

Green, P. E., \& Srinivasan, V. (1978). Conjoint analysis in consumer research: Issues and outlook. Journal of Consumer Research, 5, 103-123.

Hackman, J., \& Oldham, G. R. (1975). Development of the job diagnostic survey. Journal of Applied Psychology, 60, 159-170.

Harris, R. J. (1983). Pinning down the equity formula.
In D. M. Messick \& K. S. Cook (Eds.), Equity theory: Psychological and sociological perspectives ( $\mathrm{pp}$. 207-241). New York: Praeger.

Helson, H. (1964). Adaptation-level theory. New York: Harper \& Row.

Himmelblau, D. M. (1972). Applied nonlinear programming. New York: McGraw-Hill.

Huppertz, J. W., Arenson, S. J., \& Evans, R. H. (1978). An application of equity theory to buyer-seller exchange situations. Journal of Marketing Research, 15, $250-260$.

Kruskal, J. B. (1978). Factor analysis and principal components, I. Bilinear models. In W. H. Kruskal \& J. M. Tanur (Eds.), International encyclopedia of statistics, Vol. I (pp. 307-330). New York: The Free Press.

LaBarbera, P. A., \& Mazursky, D. (1983). A longitudinal assessment of consumer satisfaction/dissatisfaction: The dynamic aspect of the cognitive process. Journal of Marketing Research, 20, 393-404.

Liefeld, J. P., Edgecombe, F. H. C., \& Wolfe, L. (1975). Demographic characteristics of Canadian consumer complainers. Journal of Consumer Affairs, 9, 73-89.

Luce, R. D. (1959). Individual choice behavior. New York: Wiley.

McCullagh, P., \& Nelder, J. A. (1983). Generalized linear models. New York: Chapman and Hall.

McFadden, D. (1976). Quantal choice analysis: A survey. Annals of Economic and Social Measurement, 5, 363-390.

Messick, D. M., \& Sentis, K. (1983). Faimess, preference, and fairness biases. In D. M. Messick \& K. S. Cook (Eds.), Equity theory: Psychological and sociological perspectives (pp. 61-94). New York: Plenum Press.

Nelder, J. A., \& Wedderburn, R. W. M. (1972). Generalized linear models. Journal of the Royal Statistical Society, Series A, 135, 370-384.

Noma, E., \& Johnson, J. (1977). Constraining nonmetric multidimensional scaling configurations (Technical Report 60). Ann Arbor: The University of Michigan, Human Performance Center.

Oliver, R. L. (1977). Effect of expectation and disconfirmation on postexposure product evaluations: An alternative interpretation. Journal of Applied Psychology, 62, 480-486.

Oliver, R. L. (1980). A cognitive model of the antecedents and consequences of satisfaction decisions. Journal of Marketing Research, 17, 460-469.

Pope, C. R. (1978). Consumer satisfaction in a health maintenance organization. Journal of Health \& Social Behavior, 19, 291-303.

Powell, M. J. D. (1977). Restart procedures for the conjugate gradient method. Mathematical Programming, 12, 241-254.

Rao, S. S. (1979). Optimization: Theory and applications. New York: Wiley.

Scarpello, V., \& Campbell, J. P. (1983). Job satisfac- 
tion: Are all the parts there? Personnel Psychology, $36,577-600$.

Schonemann, P. H., \& Wang, M. M. (1972). An individual difference model for the multidimensional analysis of preference data. Psychometrika, 37, 275305.

Slater, P. (1960). The analysis of personal preferences. British Journal of Statistical Psychology, 13, 119 135.

Swan, J. E., \& Trawick, I. F. (1981). Disconfirmation of expectations and satisfaction with a retail service. Journal of Retailing, 57, 49-67.

Thurstone, L. L. (1927). A law of comparative judgment. Psychological Review, 34, 273-286.

Torgerson, W. S. (1958). Theory and methods of scaling. New York: Wiley.

Tucker, L. R. (1960). Intra-individual and inter-individual multidimensionality. In $\mathrm{H}$. Gulliksen \& $\mathrm{S}$. Messick (Eds.), Psychological scaling: Theory and applications (pp. 155-167). New York: Wiley.

Ware, J. E., Jr. (1978). Effects of acquiescent response set on patient satisfaction ratings. Medical Care, 16 , 327-336.

Ware, J. E., Jr., \& Snyder, M. K. (1975). Dimensions of patient attitudes regarding doctors and medical care services. Medical Care, 13, 669-682.

Warland, R. H., Hermann, R. O., \& Willits, J. (1975).
Dissatisfied consumers: Who gets upset and who takes action? Journal of Consumer Affairs, 9, 148-163.

Weiner, B., Frieze, I., Kukla, A., Reed, L., Rest, S., \& Rosenbaum, R. M. (1971). Perceiving the causes of success and failure. Morristown NJ: General Learning Press.

Weiner, B., Russell, D., \& Lerman, D. (1978). Affective consequences of causal ascriptions. In $\mathrm{J} . \mathrm{H}$. Harvey, W. Ickes, \& R. F. Kidd (Eds.), New directions in attribution research, Vol. 2 (pp. 51-90). Hillsdale NJ: Laurence Erlbaum Associates.

Westbrook, R. A. (1980). Intrapersonal affective influences on consumer satisfaction with products. Journal of Consumer Research, 7, 49-54.

Westbrook, R. A. (1981). Sources of consumer satisfaction with retail outlets. Journal of Retailing, $3,68-$ 85.

Zinnes, J. L., \& Wolff, R. P. (1977). Single and multidimensional same-different judgments. Journal of Mathematical Psychology, 16, 30-50.

\section{Author's Address}

Send requests for reprints or further information to Wayne S. DeSarbo, The Wharton School, University of Pennsylvania, Philadelphia PA 19104, U.S.A. 